\title{
Discretization of Nonlinear Non-affine Time Delay Systems Based on Second-order Hold
}

\author{
Yuan-Liang Zhang \\ School of Mechanical Engineering, Huaihai Institute of Technology, Jiangsu Province Research and Development Institute of Marine \\ Resources, Lianyungang 222005, China
}

\begin{abstract}
When calculating the sampled-date representation of nonlinear systems second-order hold (SOH) assumption can be applied to improving the precision of the discretization results. This paper proposes a discretization method based on Taylor series and the $\mathrm{SOH}$ assumption for the nonlinear systems with the time delayed non-affine input. The mathematical structure of the proposed discretization method is explored. This proposed discretization method can provide a precise and finite dimensional discretization model for the nonlinear time-delayed non-affine system by keeping the truncation order of the Taylor series. The performance of the proposed discretization method is evaluated by doing the simulation using a nonlinear system with the time-delayed non-affine input. Different input signals, time-delay values and sampling periods are considered in the simulation to investigate the proposed method. The simulation results demonstrate that the proposed method is practical and easy for time-delayed nonlinear non-affine systems. The comparison between $\mathrm{SOH}$ assumption with first-order hold (FOH) and zero-order hold ( $\mathrm{ZOH}$ ) assumptions is given to show the advantages of the proposed method.
\end{abstract}

Keywords: Nonlinear system, non-affine input, discretization, Taylor series, second-order hold (SOH).

\section{Introduction}

Time-delay systems are also called systems with aftereffect or dead-time, hereditary systems, equations with deviating argument or differential-difference equations. Opposed to ordinary differential equations (ODEs), they belong to the class of functional differential equations (FDEs) which are infinite dimensional ${ }^{[1]}$. The analysis of the timedelay systems is one of the difficult but hot points in control theory and control engineering domain. The reasons for this can be grouped into two major kinds. The first one is that time delay is an applied problem. Time delay problem is becoming increasingly more widespread in control systems because of the convergence of communication and computational systems with traditional control engineering. Controller communication, especially communication over local-area networks (LANs) or wide-area networks (WANs), and complex computations resulting from digital controller implementations result in large time delays. Many practical systems such as automotive powertrain systems ${ }^{[2]}$, combustion systems ${ }^{[3]}$, chemical reactors ${ }^{[4,5]}$, teleoperators ${ }^{[6]}$, and master-slave manipulator systems ${ }^{[7]}$ are described by time-delay models. The second reason is that control systems with non-negligible time delays exhibit complex behaviors because of their infinite dimensionality. When expressed in the continuous time domain, even a linear time invariant system with a constant time delay in the input or state has infinite dimensionality. For continuous time sys-

Regular Paper

Manuscript received April 11, 2013; revised September 24, 2013

This paper was supported by Jiangsu Province University Natural Science Research Project (No. 13KJB510003) and Jiangsu Province Research and Development Institute of Marine Resources Science and Technology Open Fund Project (No. JSIMR11B05). tems, the time delay problems can in principle be treated by the infinite dimension system theory approach. However, it is generally not easy to understand the approach and obtain the corresponding controller which is given in terms of operator Riccati equations ${ }^{[8]}$. And controller design techniques developed over the last several decades for finite dimensional systems are difficult to apply to time delay systems. Thus it is necessary to develop a control system design scheme that resolves the time delay problem.

Since time delay often occurs in various engineering systems and causes serious deterioration of the stability and performance of the systems, considerable research has been done for the control of time delay systems ${ }^{[9]}$. $\mathrm{Ji}^{[10]}$ proposed an adaptive neural network control method for a class of perturbed strict-feedback nonlinear systems with time delays. Tang and Ding ${ }^{[11]}$ concerned with the model predictive control of networked control systems with time delay and data packets disorder. Zhu et al. ${ }^{[12]}$ dealt with the problem of tracking control for a class of high order nonlinear systems with input delay. $\mathrm{Mu}$ and $\mathrm{Gao}^{[13]}$ discussed the problem of global input-to-state stability for discrete time piecewise affine systems with time delay. Bedoui et al. ${ }^{[14]}$ proposed an approach for simultaneous online identification of the time delay and dynamic parameters of discrete time delay systems ${ }^{[14]}$. Balasubramaniam and Senthilkumar ${ }^{[15]}$ discussed the delay-dependent robust stabilization and $H_{\infty}$ control for uncertain stochastic Takagi-Sugeno fuzzy systems with discrete interval and distributed time-varying delays. Ahmida and Tissir ${ }^{[16]}$ discussed the delay-dependent exponential stability of a class of uncertain T-S fuzzy switched systems with time delay. Phat et al. ${ }^{[17]}$ addressed 
the exponential stability for a class of nonlinear hybrid timedelay systems.

In practice, nonlinear control strategies are usually implemented using a microcontroller or a digital signal processor. As a direct consequence of this, the associated control algorithms must operate using discrete time intervals. The continuous-time control law is designed based on a continuous-time system model and then is discretized. Or a continuous-time system model is discretized and then the discrete time control law is designed based on it. It should be emphasized that in both design approaches, the time discretization of either the controller or the system model is necessary. In the field of the discretization, for the time free continuous time systems the traditional numerical techniques such as Euler and Runge-Kutta method have been used to obtain the sampled-date representations. However, these methods require a small sampling interval. But due to the physical and technical limitations slow sampling is becoming inevitable. For large sample period case, the Taylor series method was used to improve the performance of the controller ${ }^{[18]}$. However, in previous papers, the zero-order hold $(\mathrm{ZOH})$ and first-order hold $(\mathrm{FOH})$ assumptions were used in the discretization method ${ }^{[19]}$. The performance of the discretization methods using these assumptions is highly dependent on the input signal, and the sampling period should be short enough for the desired control precision.

A high-order method is the one that provides increased accuracy with only a modest increase in the computational cost $^{[20]}$. Wei and $\mathrm{Liu}^{[21]}$ studied the problem of output feedback fault-tolerant control design for continuous-time linear time-invariant systems via high-order hold sampleddata controllers. Tornero and Tomizuka ${ }^{[22]}$ considered the problem of the computation of a dual-rate high-order-hold discrete equivalent controller. Lampe and Rosenwasser ${ }^{[23]}$ studied the digital control problem for a continuous-time linear time-invariant (LTI) process with generalized higherorder hold and time delay. Zhang ${ }^{[24]}$ discussed sampleddata control design problem by the first-order hold input.

This paper proposes a time discretization method for nonlinear input-driven dynamic systems with time delayed non-affine input. This method is based on the Taylor series and second-order hold ( $\mathrm{SOH})$ assumption. This discretization method can provide a finite dimensional representation, which allows the direct application of the existing nonlinear control system design techniques. Secondly, the performance evaluation of the proposed algorithm is presented using a numerical simulation with different sampling periods, time-delays and inputs, and the results are compared to those obtained by using the $\mathrm{ZOH}$ and $\mathrm{FOH}$ method.

The paper is organized as follows. Section 2 contains some mathematical preliminaries and Section 3 includes the main results of this paper, in which a new time discretization method for nonlinear systems with time delayed nonaffine input is introduced. Finally, one numerical simulation with different sampling periods, time-delays, and kinds of inputs is presented in Section 4 to demonstrate the effectiveness of the proposed discretization method, and Section 5 provides a few concluding remarks drawn from this study.

\section{Preliminaries}

In the present study the nonlinear continuous time control systems with time delayed non-affine input are considered using a state-space representation form [25]:

$$
\begin{aligned}
\dot{x}(t)= & f_{0}(x(t))+g_{1}(x(t)) u(t-D)+ \\
& g_{2}(x(t)) u(t-D)^{2}+\cdots+g_{m}(x(t)) u(t-D)^{m}
\end{aligned}
$$

and

$$
\dot{x}(t)=f(x(t), u(t-D))
$$

where $x \in \mathbf{R}^{n}$ is the vector of the states representing an open and connected set, $u \in \mathbf{R}$ is the input variable, $m$ is an integer which indicates the order of the input $u$, and $D$ is the system constant time-delay (dead-time) that directly affects the input. It is assumed that $f_{0}: \mathbf{R}^{n} \rightarrow \mathbf{R}^{n}, g_{i}: \mathbf{R}^{n} \rightarrow \mathbf{R}^{n}$, $i=1,2, \cdots, m$ and $f: \mathbf{R}^{n} \times \mathbf{R} \rightarrow \mathbf{R}^{n}$ are smooth mappings.

An equidistant grid on the time axis with mesh $T=$ $t_{k+1}-t_{k}>0$ is considered where sampling interval is $\left[t_{k}, t_{k+1}\right)=[k T,(k+1) T)$ and $T$ is the sampling period. Furthermore, we suppose the time-delay $D$ and mesh $T$ are related as follows

$$
D=q T+\gamma
$$

where $q \in\{0,1,2, \cdots\}$ and $0 \leqslant \gamma<T$. That is, the timedelay $D$ is customarily represented as an integer multiple of the sampling period adding a fractional part of $T^{[26]}$. In this paper, it is also assumed that system (1) is driven by an input that is piecewise quadratic over the sampling interval, i.e., the $\mathrm{SOH}$ assumption holds true.

For the $\mathrm{ZOH}$ assumption, it is assumed that system (1) is driven by an input that is piecewise constant over the sampling interval. And for the FOH assumption, it is assumed that the original piecewise continuous input function is approximated by a piecewise linear one over the sampling interval. Let us consider $\mathrm{ZOH}, \mathrm{FOH}$ and $\mathrm{SOH}$ assumptions in more detail.

Under the $\mathrm{ZOH}$ assumption, in the delay free case, while $D=0$, we have

$$
u_{Z}(t)=u(k)=\text { constant, for } k T \leqslant t<k T+T
$$

where $u(k)=u(k T)$. Based on the above notation one can deduce that for $D>0$ the delayed input variable attains the following two distinct values within the sampling interval:

$$
u_{Z}(t-D)= \begin{cases}u(k-q-1), & \text { if } k T \leqslant t<k T+\gamma, \\ u(k-q), & \text { if } k T+\gamma \leqslant t<k T+T\end{cases}
$$

Here, subscript $Z$ denotes that input approximation is performed under the $\mathrm{ZOH}$ assumption. 
Under the FOH assumption, while $D=0$, we have $u_{F}(t)=u(k)+v(k)(t-k T), \quad$ for $k T \leqslant t<k T+T$.

Here, subscript $F$ denotes that input approximation is performed under the FOH assumption, and in what follows:

$$
\begin{aligned}
& v(k)=\frac{u((k+1) T)-u(k T)}{T} \quad \text { or } \\
& v(k)=\left.\frac{\mathrm{d} u(t)}{\mathrm{d} t}\right|_{t=k T}, \quad k=0,1,2, \cdots
\end{aligned}
$$

For $D>0$, it is rather straightforward to verify that the delayed input variable attains the following values within the sampling interval.

$$
\begin{aligned}
& u_{F}(t-D) \equiv \\
& \left\{\begin{array}{c}
u(k-q-1)+v(k-q-1)[t-k T+(T-\gamma)], \\
t \in[k T, k T+\gamma) \\
u(k-q)+v(k-q)[t-k T-\gamma], \\
t \in[k T+\gamma, k T+T) .
\end{array}\right.
\end{aligned}
$$

Under the $\mathrm{SOH}$ assumption and in the case of delay free case, in the time interval $k T \leqslant t<k T+T$ the input can be expressed as follows.

$$
\begin{aligned}
u(t)= & u(k)+\frac{u(k)-u(k-1)}{T}(t-k T)+ \\
& \frac{1}{2} \frac{u(k)-2 u(k-1)+u(k-2)}{T^{2}}(t-k T)^{2} .
\end{aligned}
$$

Furthermore, let

$$
a(k)=\frac{v(k)-v(k-1)}{T}
$$

where $a(k)$ represents the second-order derivation at the instant $k T$.

Equation (8) is shortly represented as

$$
u(t)=u(k)+v(k)(t-k T)+\frac{1}{2} a(k)(t-k T)^{2} .
$$

In the case of $D>0$, we have

$$
\begin{aligned}
& u(t-D) \equiv \\
& \left\{\begin{array}{l}
u(k-q-1)+v(k-q-1)[t-k T+(T-\gamma)]+ \\
\frac{1}{2} a(k-q-1)[t-k T+(T-\gamma)]^{2}, \quad t \in[k T, k T+\gamma) \\
u(k-q)+v(k-q)[t-k T-\gamma]+ \\
\frac{1}{2} a(k-q)[t-k T-\gamma]^{2}, \quad t \in[k T+\gamma, k T+T) .
\end{array}\right.
\end{aligned}
$$

\section{Discretization of nonlinear systems with time delayed non-affine input}

\subsection{Discretization in the case of the delay free input}

At this point, it would be methodologically appropriate to succinctly present and delineate the time-discretization method available for delay-free $(D=0)$ nonlinear control systems. The ensuing brief description of the time discretization method for delay-free nonlinear systems will serve as a natural point of departure for the development of a discretization method for nonlinear systems with time delayed non-affine input.

When $D=0$, within the sampling interval and under the $\mathrm{SOH}$ assumption, the solution of system (1) is expanded into a uniformly convergent Taylor series:

$$
x(k+1)=x(k)+\sum_{l=1}^{\infty} A^{[l]}(x(k), u(k), v(k), a(k)) \frac{T^{l}}{l !}
$$

where $A^{[l]}(x, u, v, a)$ are determined recursively by

$$
\begin{aligned}
A^{[1]}(x, u, v, a)= & f(x, u) \\
A^{[2]}(x, u, v, a)= & \frac{\partial A^{[1]}(x, u, v, a)}{\partial x} A^{[1]}(x, u, v, a)+ \\
& \frac{\partial A^{[1]}(x, u, v, a)}{\partial u} v \\
A^{[l+2]}(x, u, v, a)= & \frac{\partial A^{[l+1]}(x, u, v, a)}{\partial x} A^{[1]}(x, u, v, a)+ \\
& \frac{\partial A^{[l+1]}(x, u, v, a)}{\partial u} v+\frac{\partial A^{[l]}(x, u, v, a)}{\partial u} a
\end{aligned}
$$

where $l=1,2,3, \cdots$. Notice that the resulting coefficients $A^{[l]}(x(k), u(k), v(k), a(k))$ can be easily computed by taking successive partial derivatives of the right hand-side of (1).

Therefore, an exact sampled-data representation (ESDR) of system (1) can be derived by retaining the full infinite series of (12), namely

$$
\begin{aligned}
x(k+1)= & \Phi_{T}(x(k), u(k), v(k), a(k))= \\
& x(k)+\sum_{l=1}^{\infty} A^{[l]}(x(k), u(k), v(k), a(k)) \frac{T^{l}}{l !} .
\end{aligned}
$$

Simultaneously, an approximate sampled-data representation (ASDR) of (1) is obtained from a truncation of the Taylor series order $N$, i.e.,

$$
\begin{aligned}
x^{N}(k+1)= & \Phi_{T}^{N}\left(x^{N}(k), u(k), v(k), a(k)\right)= \\
& x^{N}(k)+\sum_{l=1}^{N} A^{[l]}\left(x^{N}(k), u(k), v(k), a(k)\right) \frac{T^{l}}{l !}
\end{aligned}
$$

where subscript $T$ of mapping $\Phi_{T}^{N}$ denotes the dependence on the sampling period, and superscript $N$ denotes the finite series truncation order associated with the ASDR of (15).

\subsection{Discretization in the case of the time delayed non-affine input}

When $D>0$, by applying the Taylor discretization method for system (1) over the subinterval $[k T, k T+\gamma)$ and taking into account (11), one can obtain the state vector evaluated at $k T+\gamma$ as a function of $x(k), u(k-q-1)$, 


$$
\begin{aligned}
& v(k-q-1) \text { and } a(k-q-1) . \\
& x(k T+\gamma)=x(k)+ \\
& \sum_{l=1}^{\infty} A^{[l]}(x(k), u(k-q-1), v(k-q-1), a(k-q-1)) \frac{\gamma^{l}}{l !} .
\end{aligned}
$$

Similarly, the application of the Taylor discretization method to the $[k T+\gamma, k T+T)$ subinterval yields the state vector evaluated at $(k+1) T$ as a function of $x(k T+\gamma)$, $u(k-q), v(k-q)$ and $a(k-q)$.

$$
\begin{aligned}
& x(k+1)=x(k T+\gamma)+ \\
& \sum_{l=1}^{\infty} A^{[l]}(x(k T+\gamma), u(k-q), v(k-q), a(k-q)) \frac{(T-\gamma)^{l}}{l !} .
\end{aligned}
$$

The ASDR of (16) and (17) are obtained from the truncation of the Taylor series order $N$, as shown below,

$$
\begin{aligned}
& x^{N}(k T+\gamma)=x^{N}(k)+ \\
& \sum_{l=1}^{N} A^{[l]}\left(x^{N}(k), u(k-q-1), v(k-q-1), a(k-q-1)\right) \frac{\gamma^{l}}{l !} \\
& x^{N}(k+1)=x^{N}(k T+\gamma)+ \\
& \sum_{l=1}^{N} A^{[l]}\left(x^{N}(k T+\gamma), u(k-q), v(k-q), a(k-q)\right) \frac{(T-\gamma)^{l}}{l !} .
\end{aligned}
$$

The subsequent calculation of the corresponding Taylor coefficients $A^{[l]}(x, u, v, a)$ can be realized by using (13).

Theorem 1. Let $x^{0}$ be an equilibrium point of the original non-affine input nonlinear continuous-time system:

$$
\dot{x}=f(x(t), u(t))
$$

that belongs to the equilibrium manifold:

$$
E^{c}=\left\{x \in \mathbf{R}^{n} \mid \exists u \in \mathbf{R}: f(x, u)=0\right\}
$$

and $u=u^{0}$ be the corresponding equilibrium value of the input variables: $f\left(x^{0}, u^{0}\right)=0,\left.\frac{\mathrm{d} u}{\mathrm{~d} t}\right|_{u_{0}}=0$ and $\left.\frac{\mathrm{d}^{2} u}{\mathrm{~d} t^{2}}\right|_{u_{0}}=0$. Then $x^{0}$ belongs to the equilibrium manifold: $E^{d}=\{x \in$ $\left.\mathbf{R}^{n} \mid \exists u \in \mathbf{R}: \Phi_{T}^{D}(x, u)=x\right\}$ of the ESDR: $x(k+1)=$ $\Phi_{T}^{D}(x(k), u(k-q-1), u(k-q), v(k-q-1), v(k-q), a(k-$ $q-1), a(k-q))$ and ASDR: $x(k+1)=\Phi_{T}^{N, D}(x(k), u(k-$ $q-1), u(k-q), v(k-q-1), v(k-q), a(k-q-1), a(k-q))$ obtained under the proposed Taylor discretization method, with $u=u^{0}$ being the corresponding equilibrium values of the input variables: $\Phi_{T}^{D}\left(x^{0}, u^{0}\right)=x^{0}$ and $\Phi_{T}^{N, D}\left(x^{0}, u^{0}\right)=$ $x^{0}$.

Proof. $x^{0}$ is the equilibrium point and $u^{0}$ is the corresponding equilibrium values of the input variables $\Rightarrow A^{[1]}\left(x^{0}, u^{0}\right)=f\left(x^{0}, u^{0}\right)=0, A^{[2]}\left(x^{0}, u^{0}, v^{0}, a^{0}\right)=$ $\frac{\partial A^{[1]}\left(x^{0}, u^{0}, v^{0}, a^{0}\right)}{\partial x} A^{[1]}\left(x^{0}, u^{0}\right)+\frac{\partial A^{[1]}\left(x^{0}, u^{0}, v^{0}, a^{0}\right)}{\partial u} v^{0}=0 \Rightarrow$ $A^{[l+2]}\left(x^{0}, u^{0}, v^{0}, a^{0}\right)=\frac{\partial A^{[l+1]}\left(x^{0}, u^{0}, v^{0}, a^{0}\right)}{\partial x} A^{[1]}\left(x^{0}, u^{0}\right)+$ $\frac{\partial A^{[l+1]}\left(x^{0}, u^{0}, v^{0}, a^{0}\right)}{\partial u} v^{0}+\frac{\partial A^{[l]}\left(x^{0}, u^{0}, v^{0}, a^{0}\right)}{\partial u} a^{0}=0$, for all $l \in\{1,2,3, \cdots\} \Rightarrow \Phi_{\gamma}\left(x^{0}, u^{0}, v^{0}, a^{0}\right)=x^{0}+$ $\sum_{l=1}^{\infty}\left(A^{[l]}\left(x^{0}, u^{0}, v^{0}, a^{0}\right) \frac{\gamma^{l}}{l !}\right)=x^{0}, \Phi_{T}^{D}\left(x^{0}, u^{0}, v^{0}, a^{0}\right)=$ $\Phi_{T-\gamma}\left(\Phi_{\gamma}\left(x^{0}, u^{0}, v^{0}, a^{0}\right), u^{0}, v^{0}, a^{0}\right)=x^{0}$.

Similar arguments apply to the $\Phi_{T}^{N, D}$ map of the ASDR. Therefore, $x^{0}$ belongs to the equilibrium manifold $E^{d}$ of the ESDR and ASDR for any finite truncation order $N$. That is, the proposed discretization method does not change the equilibrium point of the original non-affine input nonlinear continuous-time system.

\section{Simulation}

The performance of the proposed time discretization method is evaluated by applying it to a nonlinear system with the time delayed non-affine input. Reference solutions for the system are required to validate the proposed time discretization method. In this paper, the Matlab ODE solver is used to obtain the reference solutions. The discrete values obtained at every time step using the proposed time discretization method are compared to the values obtained using the Matlab ODE solver at the corresponding time steps. The partial derivative terms involved in the Taylor series expansion are determined recursively. In the simulation, these partial derivative terms are calculated using Maple.

The system considered in this paper is assumed to be a nonlinear control system ${ }^{[27]}$.

$$
\begin{aligned}
& \dot{x}_{1}=-x_{1}^{3}+x_{1} \mathrm{e}^{x_{2}} u(t-D)^{2} \\
& \dot{x}_{2}=x_{2}^{2} u(t-D) .
\end{aligned}
$$

Since for system (22) $N=3$ is good enough for getting the accurate discretization results, in the simulation the truncation of the Taylor series order $N$ is chosen as 3 . First we compare the proposed discretization method with the traditional Euler method. In this case, the parameters are chosen as $x_{1}(0)=1.0, x_{2}(0)=-1.0, T=0.2 \mathrm{~s}$ and $D=0.32 \mathrm{~s}$. In this case $q=1$ and $\gamma=0.12 \mathrm{~s}$. The input is assumed to be a constant one, $u=1.5$. The results obtained by the Matlab ODE solver and the proposed discretization method with $\mathrm{SOH}$ assumption are shown in Fig. 1. Fig. 2 shows the errors of states $x_{1}$ and $x_{2}$ by using the proposed discretization method with $\mathrm{SOH}$ assumption and the traditional Euler method, respectively. From Fig. 2, it can be seen that the proposed discretization method can provide better discretization results than the traditional Euler method.

Secondly, we choose the parameters as $x_{1}(0)=1.0$, $x_{2}(0)=-1.0, T=0.04 \mathrm{~s}$ and $D=0.018 \mathrm{~s}$. In this case $q=0$ and $\gamma=0.018 \mathrm{~s}$. The input is assumed to be a constant one, $u=1.2$. The results obtained by the Matlab ODE solver and the proposed discretization method with $\mathrm{SOH}$ assumption are shown in Fig. 3. Fig. 4 shows the errors of states $x_{1}$ and $x_{2}$ in the case of $\mathrm{ZOH}, \mathrm{FOH}$ and $\mathrm{SOH}$ assumptions, respectively. In this case, since the input $u$ is a constant one the performance of cases of $\mathrm{ZOH}, \mathrm{FOH}$ and $\mathrm{SOH}$ assumptions is similar. 

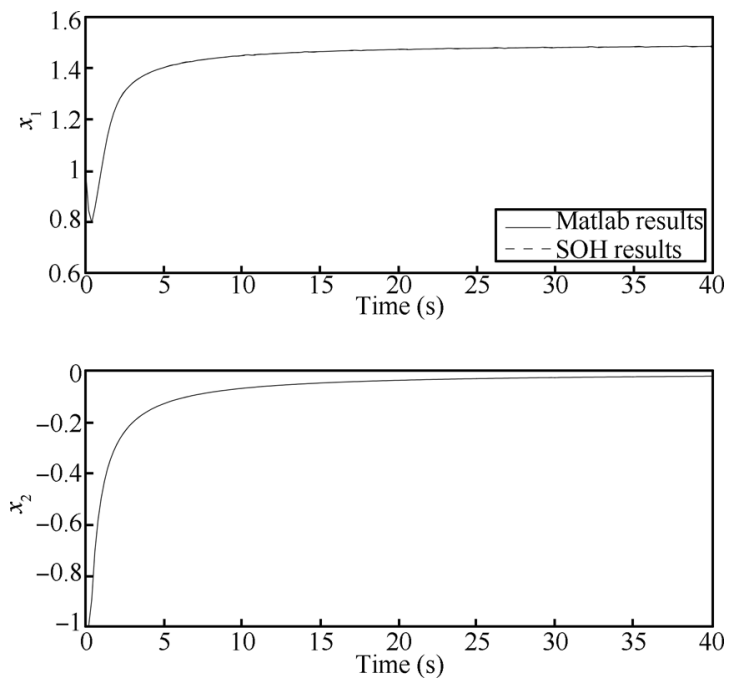

Fig. 1 The discretization results for Case 1
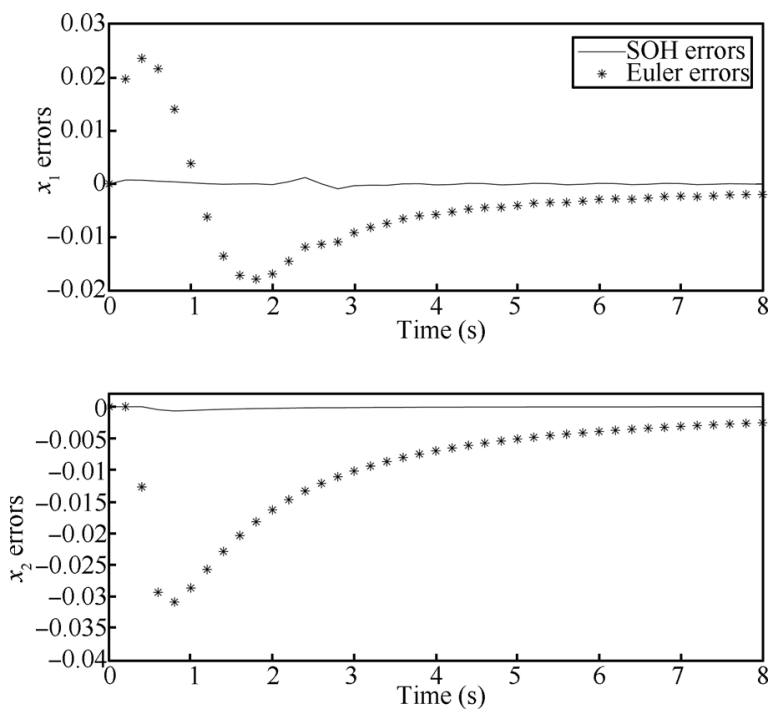

Fig. 2 State errors of $\mathrm{SOH}$ and Euler method for Case 1

Then we choose the parameters as $x_{1}(0)=1.0, x_{2}(0)=$ $-1.0, T=0.05 \mathrm{~s}, D=0.22 \mathrm{~s}, u=\sin (1.5 t) ; T=0.1 \mathrm{~s}, D=$ $0.56 \mathrm{~s}, u=0.05 t^{2}$; and $T=0.2 \mathrm{~s}, D=0.32 \mathrm{~s}, u=0.05 t^{2}$. In these three cases $q=4, \gamma=0.02 \mathrm{~s} ; q=5, \gamma=0.06 \mathrm{~s}$; and $q=1, \gamma=0.12 \mathrm{~s}$, respectively. The results obtained by the Matlab ODE solver and the proposed discretization method with $\mathrm{SOH}$ assumption of these three cases are shown in Figs. 5, 7 and 9, respectively. Figs. 6, 8 and 10 show the errors of the state in the cases of $\mathrm{ZOH}, \mathrm{FOH}$ and $\mathrm{SOH}$ assumptions of these three cases, respectively.

From the simulation results, it can be seen that the proposed time discretization method with $\mathrm{SOH}$ assumption can provide satisfied discretization results for nonlinear systems with time delayed non-affine input. And in these three cases the inputs are not the constant ones. From Figs. 6, 8 and 10 , it can be seen that when the input is not a constant one the Taylor discretization method with SOH assumption performs better than the methods with $\mathrm{FOH}$ and $\mathrm{ZOH}$ assumptions. And in the cases $1-3$, the simulation time is
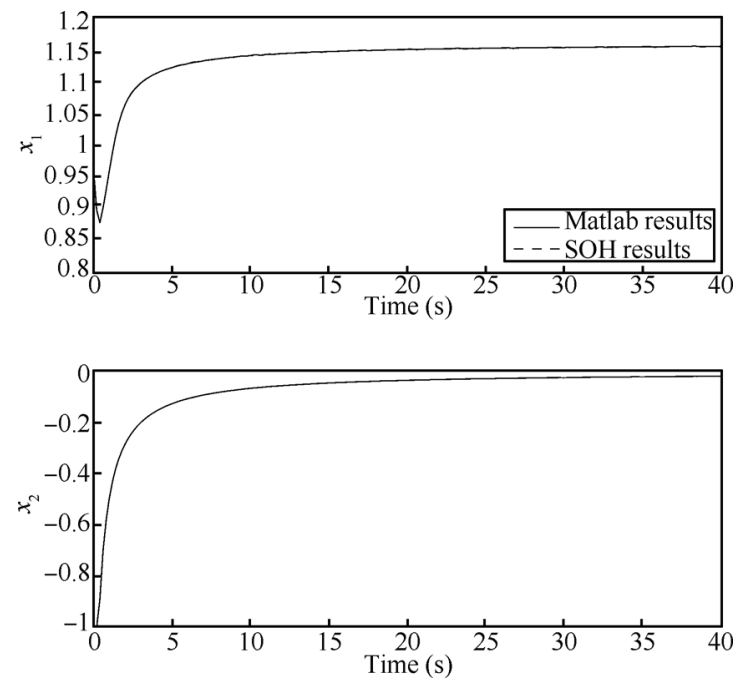

Fig. 3 The discretization results for Case 2
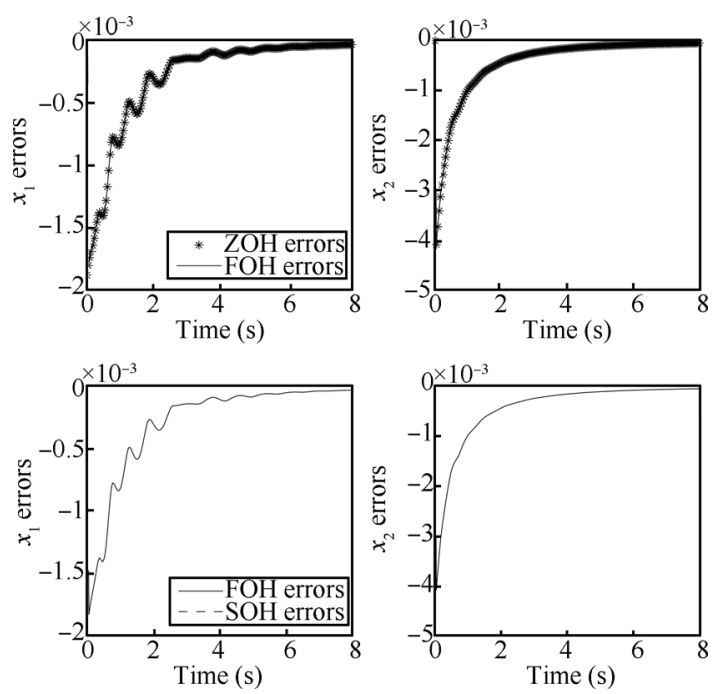

Fig. 4 State errors of $\mathrm{ZOH}, \mathrm{FOH}$ and $\mathrm{SOH}$ for Case 2
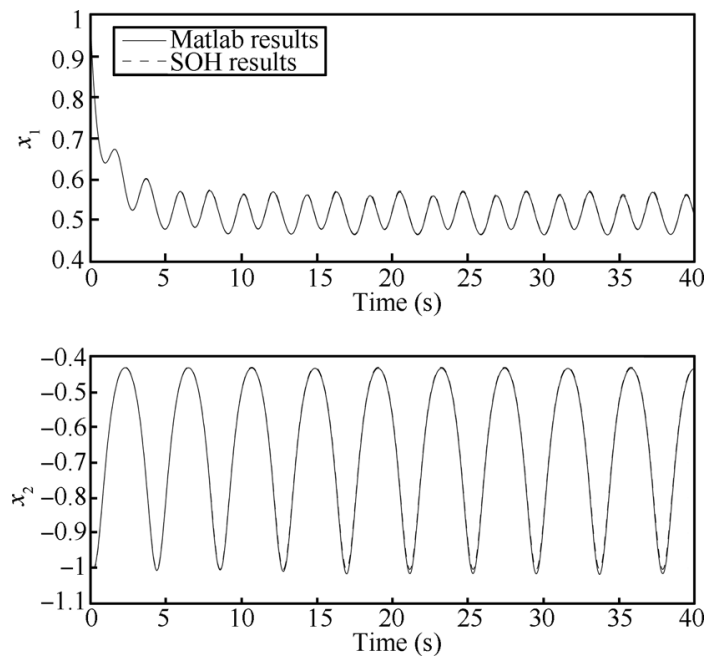

Fig. 5 The discretization results for Case 3 

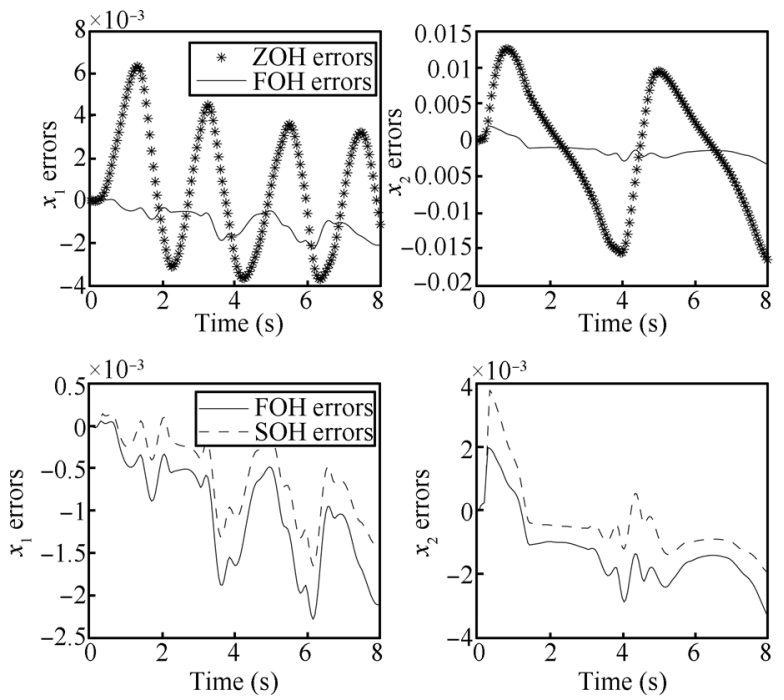

Fig. 6 State errors of $\mathrm{ZOH}, \mathrm{FOH}$ and $\mathrm{SOH}$ for Case 3
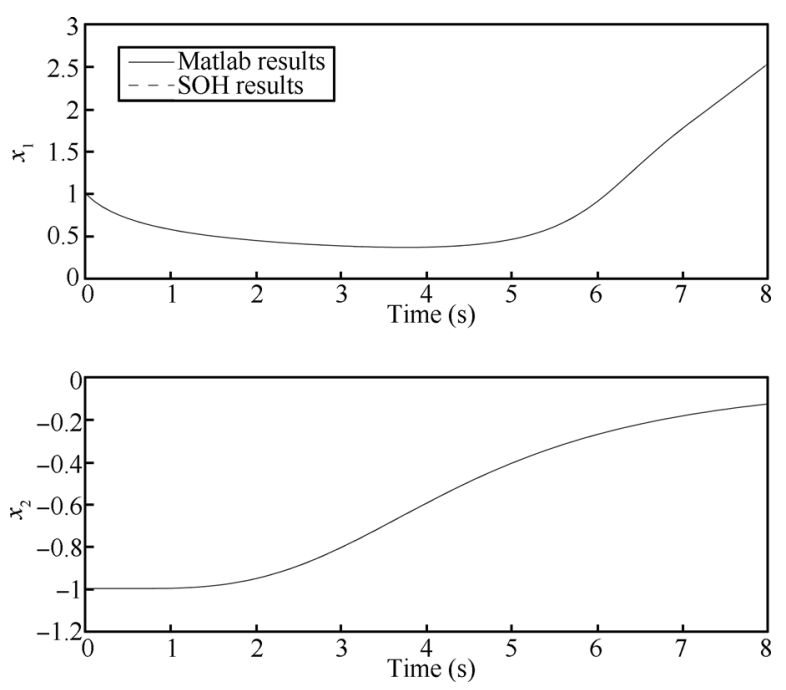

Fig. 7 The discretization results for Case 4

long enough to show the system's steady-state response. Figs. 1, 3 and 5 show that the proposed time discretization method does not change the original system's stability.

The computational costs are considered at the end of this section, where Table 1 shows the computing times used to get the discretization results of the proceeding simulations using Taylor series with the $\mathrm{SOH}$ assumption, FOH assumption and the $\mathrm{ZOH}$ assumption, respectively. The computing time is calculated on a computational process with 5000 steps. It can be seen from Table 1 that the computing times using the Taylor series with the $\mathrm{SOH}$ assumption are moderately longer than those when we use FOH and $\mathrm{ZOH}$ assumptions but the accuracy is much better for the process with $\mathrm{SOH}$ assumption.

Table 1 Computing times for the simulations

\begin{tabular}{cccc}
\hline Computing time (s) & SOH & FOH & ZOH \\
\hline Case 2 & 6.47 & 5.84 & 4.46 \\
Case 3 & 8.65 & 7.51 & 5.87 \\
Case 4 & 6.31 & 5.91 & 4.42 \\
Case 5 & 7.02 & 6.21 & 5.01 \\
\hline
\end{tabular}
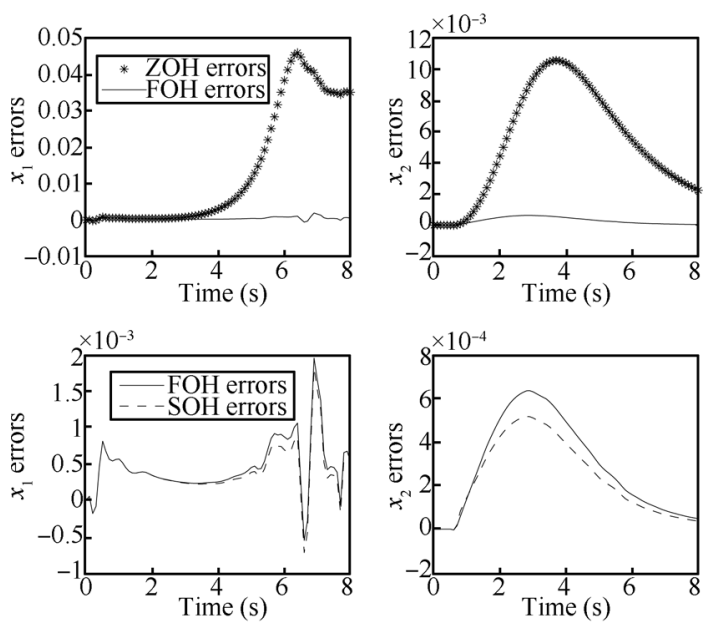

Fig. 8 State errors of $\mathrm{ZOH}, \mathrm{FOH}$ and $\mathrm{SOH}$ for Case 4
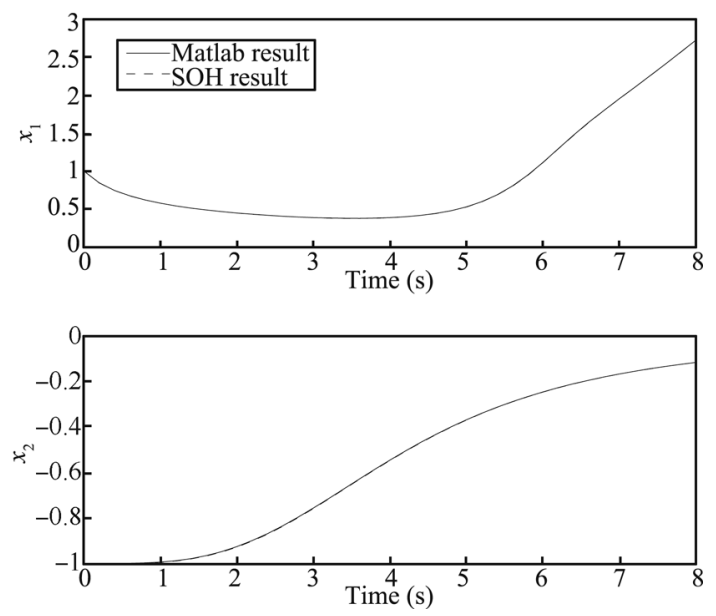

Fig. 9 The discretization results for Case 5
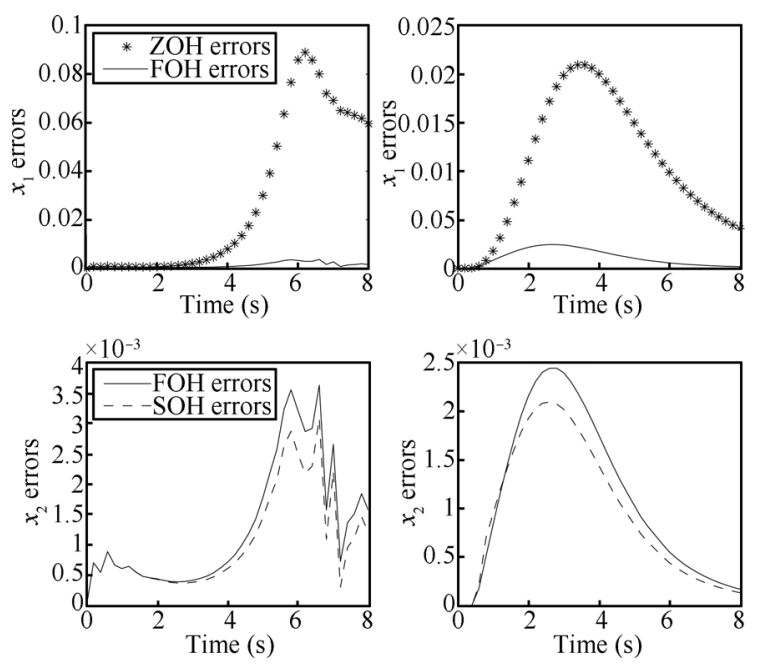

Fig. 10 State errors of $\mathrm{ZOH}, \mathrm{FOH}$ and $\mathrm{SOH}$ for Case 5

\section{Conclusion}

A scheme based on the Taylor series combined with the $\mathrm{SOH}$ assumption is proposed for the derivation of a discretetime representation of the nonlinear systems with time de- 
layed non-affine input. The mathematical structure of the new discretization scheme is explored. The derived time discretization method provides a finite-dimensional representation for nonlinear control systems with time delayed non-affine input, thereby enabling the application of existing nonlinear controller design techniques for such systems. The effect of sampling on system equilibrium property of nonlinear systems with time delayed non-affine input is examined. The performance of the proposed time discretization method is evaluated using a nonlinear system with time delayed non-affine input. In order to verify the accuracy of the proposed method the simulation results are compared with those given by Matlab ODE solver. At the same time, some comparisons are made between the method using $\mathrm{SOH}$ assumption with the traditional Euler method, and those using $\mathrm{FOH}$ and $\mathrm{ZOH}$ assumptions. The results show that the discretization method using $\mathrm{SOH}$ assumption is better than the traditional Euler method and is also better than the methods using $\mathrm{FOH}$ and $\mathrm{ZOH}$ assumptions when the input is not a constant one. Finally, the simulation results show that the proposed discretization method does not change the original system stability nor increase much computational burden.

\section{References}

[1] J. P. Richard. Time-delay systems: An overview of some recent advances and open problems. Automatica, vol. 39, no. 10, pp. 1667-1694, 2003.

[2] S. Yi, A. G. Ulsoy, P. W. Nelson. Design of observer-based feedback control for time-delay systems with application to automotive powertrain control. Journal of the Franklin Institute, vol. 347, no. 1, pp. 358-376, 2010.

[3] M. Reyes, F. V. Tinaut, C. Andrés, A. Pérez. A method to determine ignition delay times for Diesel surrogate fuels from combustion in a constant volume bomb: Inverse Livengood-Wu method. Fuel, vol. 102, pp. 289-298, 2012.

[4] W. Q. Huang, X. X. Li, S. Y. Yang, Y. Qian. Dynamic flexibility analysis of chemical reaction systems with time delay: Using a modified finite element collocation method. Chemical Engineering Research and Design, vol. 89, no. 10, pp. 1938-1946, 2011.

[5] C. I. Pop, C. M. Ionescu, R. De Keyser. Time delay compensation for the secondary processes in a multivariable carbon isotope separation unit. Chemical Engineering Science, vol. 80, pp. 205-218, 2012.

[6] J. H. Ryu, J. Artigas, C. Preusche. A passive bilateral control scheme for a teleoperator with time-varying communication delay. Mechatronics, vol. 20, no. 7, pp. 812-823, 2010.

[7] A. Forouzantabar, H. A. Talebi, A. K. Sedigh. Bilateral control of master-slave manipulators with constant time delay. ISA Transactions, vol. 51, no. 1, pp. 74-80, 2012.

[8] H. S. Zhang, G. R. Duan, L. H. Xie. Linear quadratic regulation for linear time-varying systems with multiple input delays. Automatica, vol. 42, no. 9, pp. 1465-1476, 2006.
[9] Z. C. Zhang, Y. Q. Wu. Globally asymptotic stabilization for nonlinear time-delay systems with ISS inverse dynamics. International Journal of Automation and Computing, vol. 9, no. 6, pp. 634-640, 2012.

[10] G. Ji. Adaptive neural network dynamic surface control for perturbed nonlinear time-delay systems. International Journal of Automation and Computing, vol. 9, no. 2, pp. 135-141, 2012.

[11] X. M. Tang, B. C. Ding. Design of networked control systems with bounded arbitrary time delays. International Journal of Automation and Computing, vol. 9, no. 2, pp. 182-190, 2012.

[12] Q. Zhu, A. G. Song, T. P. Zhang, Y. Q. Yang. Fuzzy adaptive control of delayed high order nonlinear systems. International Journal of Automation and Computing, vol. 9, no. 2, pp. 191-199, 2012.

[13] X. W. Mu, Y. Gao. The analysis of global input-to-state stability for piecewise affine systems with time-delay. International Journal of Automation and Computing, vol.9, no. 2, pp. 211-214, 2012.

[14] S. Bedoui, M. Ltaief, K. Abderrahim. New results on discrete-time delay systems identification. International Journal of Automation and Computing, vol.9, no. 6, pp. 570-577, 2012.

[15] P. Balasubramaniam, T. Senthilkumar. Delay-dependent robust stabilization and $H_{\infty}$ control for uncertain stochastic T-S fuzzy systems with discrete interval and distributed time-varying delays. International Journal of Automation and Computing, vol. 10, no. 1, pp. 18-31, 2013.

[16] F. Ahmida, E. H. Tissir. Exponential stability of uncertain T-S fuzzy switched systems with time delay. International Journal of Automation and Computing, vol. 10, no. 1, pp. 32-38, 2013.

[17] V. N. Phat, T. Botmart, P. Niamsup. Switching design for exponential stability of a class of nonlinear hybrid timedelay systems. Nonlinear Analysis: Hybrid Systems, vol. 3, no. 1, pp. 1-10, 2009.

[18] Y. L. Zhang, K. T. Chong. Time-discretization of time delayed non-affine system via Taylor-Lie series using scaling and squaring technique. International Journal of Control, Automation, and Systems, vol. 4, no. 3, pp. 293-301, 2006.

[19] Y. L. Zhang, O. Kostyukova, K. T. Chong. A new timediscretization for delay multiple-input nonlinear systems using the Taylor method and first order hold. Discrete Applied Mathematics, vol. 159, no. 9, pp. 924-938, 2011.

[20] S. D. Gedney, J. Ottusch, P. Petre, J. Visher, S. Wandzura. Efficient high-order discretization schemes for integral equation methods. In Proceedings of the 1997 Digest Antennas and Propagation Society International Symposium, IEEE, Montreal, Quebec, Canada, pp. 1814-1817, 1997.

[21] H. F. Wei, H. W. Liu. Output feedback fault-tolerant control for continuous-time LTI systems via high-order hold sampled-data controllers. In Proceedings of 2009 Chinese Control and Decision Conference, IEEE, Guilin, China, pp. 5318-5323, 2009. 
[22] J. Tornero, M. Tomizuka. Dual-rate high order hold equivalent controllers. In Proceedings of the 2000 American Control Conference, IEEE, Chicago, IL, USA, pp. 175-179, 2000.

[23] B. P. Lampe, E. N. Rosenwasser. Modal control for sampled-data systems with generalized higher-order hold and time delay. In Proceedings of 2006 IFAC Workshop on Time Delay Systems, IFAC, LAquila, Italy, pp. 314-319, 2006.

[24] Y. L. Zhang. Discretization of nonlinear non-affine time delay system using first order hold assumption with scaling and squaring technique. International Review on Computers and Software, vol. 7, no. 4, pp. 1860-1865, 2012.

[25] S. Yamagishi, Y. Uchida, J. Yoneyama. Output feedback stabilization of sampled-data systems by first-order hold input. In Proceedings of 2011 SICE Annual Conference, IEEE, Tokyo, Japan, pp. 283-287, 2011.

[26] D. Nesić, A. R. Teel. Sampled-data control of nonlinear systems: an overview of recent results. Lecture Notes in Con- trol and Information Sciences, vol.268, Springer, London, pp. 221-239, 2001.

[27] W. Lin. Feedback stabilization of general nonlinear control systems: a passive system approach. Systems \& Control Letters, vol. 25, no. 1, pp. 41-52, 1995.

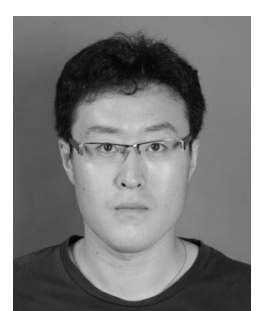

Yuan-Liang Zhang graduated from Tsinghua University, China in 2001. He received the M. Sc. degree and the Ph. D. degree from the Chonbuk National University, South Korea in 2006 and 2009, respectively. He is currently an associate professor in the School of Mechanical Engineering at Huaihai Institute of Technology, China.

His research interests include nonlinear system discretization and control and robotics.

E-mail: newlion79@gmail.com 